\section{P5.068 ROLE OF TYPE SPECIFIC HERPES SIMPLEX VIRUS SEROLOGY IN DIAGNOSIS OF PRIMARY AND RECURRENT GENITAL HERPES- A STUDY IN THE INDIAN POPULATION}

doi:10.1136/sextrans-2013-051184.1112

V Patwardhan, P Bhalla, V K Garg, A Chakravarti, K Sardana, D Rawat, S Sethi, T Bharara. Maualana Azad Medical College, New Delhi, India

Background Type specific serologic tests for herpes simplex virus (HSV)-1\&2 are used for sero-epidemiological studies. Its early application has also been shown to be of benefit in testing for primary \& recurrent infections in diagnosis and patient management. The present study was conducted to evaluate the role of type specific HSV-1\&2 antibody detection as a diagnostic modality in patients with clinically suspected genital herpes.

Methods Sera obtained from 44 patients with clinically suspected genital herpes attending the STD clinic of our institution over last 7 months were tested for type specific HSV-1\&2 IgG antibody by glycoprotein G based ELISA (HerpeSelect 1\&2, Focus Diagnostics, USA). Direct Fluorescent Antibody testing (DFA) (BIO-RAD laboratories, USA) was used for identification and typing of HSV-1\& 2 in genital ulcer specimens in all cases.

Results There were 21 cases of primary \& 23 cases of recurrent genital ulceration. DFA was positive in $68 \%$ samples (43\% only HSV-2, $9 \%$ only HSV-1, 16\% both HSV-1\&2). On comparison with DFA, the sensitivity, specificity, positive and negative predictive value of HSV-2 IgG ELISA in primary herpetic ulcer was $8 \%, 75 \%$, $33 \%, 33 \%$, while it was $79 \%, 89 \%, 92 \%, 73 \%$ in recurrent genital ulceration. The sensitivity, specificity, positive predictive and negative predictive values of HSV-1 IgG ELISA in primary herpetic ulcer was $50 \%, 67 \%, 37.5 \%$, $77 \%$, while it was $80 \%, 39 \%, 27 \%$, and $87.5 \%$ in recurrent genital ulceration.

Conclusion Our results highlight the importance of HSV-2 IgG detection in strengthening the diagnosis of recurrent HSV-2 disease, while absence of HSV-2 IgG antibody helps in excluding genital herpes as a likely cause of recurrent genital ulceration. The identification of HSV-1 IgG antibody may not be useful for diagnosis in patients of genital ulcer disease highlighting the need for typing HSV-1 strains from the genital lesion.

\section{P5.069 COPAN ESWAB ${ }^{\text {TM }}$, A LIOUID BASED MICROBIOLOGY DEVICE, CAN BE USED FOR THE PRESERVATION OF NEISSERIA GONORRHOEAE FOR CULTURE AND FOR DETECTION OF CT/NG BY GEN-PROBE ${ }^{\circledR}$ APTIMA ${ }^{\circledR}$ COMBO 2 ASSAY}

doi:10.1136/sextrans-2013-051184.1113

${ }^{1} \mathbf{M}$ Jett-Goheen, 'M Barnes, ${ }^{2} \mathrm{D}$ Duncan, ${ }^{1} \mathrm{~N}$ Quinn, ${ }^{1} \mathrm{~K}$ Ghanem, ${ }^{1} \mathrm{C}$ Gaydos. ' Johns Hopkins University, Baltimore, MD, United States; ${ }^{2}$ Baltimore City Health Department STD Clinic, Baltimore, MD, United States

Background Copan Liquid Amies Elution Swab (ESwab ${ }^{\mathrm{TM}}$ ) Collection and Transport System incorporates maintenance medium which can sustain the viability of Neisseria gonorrhoeae (NG). Swab specimens placed in the tube and stored at room temperature $\left(\mathrm{RT}^{\circ}\right)$ can be cultured within 24 hours, and aliquots for nucleic acid amplification tests (NAATs) can be processed within five days.

Methods Clinicians at a Baltimore City Health Department STD clinic used standard-of-care aluminium shaft swabs with polyester tips to collect urethral swabs from men, smeared a slide for Gram stain, and placed NG positive (by smear) swabs into investigational $\mathrm{ESwab}^{\mathrm{TM}}$ tubes. The ESwab ${ }^{\mathrm{TM}}$ collection swab applicator was then broken off into the tube and the cap was closed. Culture was performed after tube sat from 5 to 28 hours at $\mathrm{RT}^{\circ}$. Tube was vortexed for 5 seconds; $100 \mu \mathrm{l}$ was inoculated to MTM plate, streaked for isolation, and incubated in appropriate conditions for 48-72 hours;
$100 \mu \mathrm{l}$ was placed into Gen-Probe ${ }^{\circledR}$ Unisex transport tube for testing by APTIMA ${ }^{\circledR}$ COMBO 2 assay.

Results Of 35 swabs, 26 (74\%) were culture positive for presumptive NG (Gram negative diplococci, oxidase positive). Colony counts ranged from 1 to $>500$ colonies.

$35(100 \%)$ swabs were positive for NG by Gen-Probe ${ }^{\circledR}$ APTIMA $^{\circledR}$ COMBO 2 assay. Six (17\%) were also positive for Chlamydia trachomatis (CT) by Gen-Probe ${ }^{\circledR}$ assay.

Conclusion Although quality of collected clinical specimen and quantity of NG on the swab are significant variables in obtaining reliable culture results, the ESwab ${ }^{\mathrm{TM}}$ System was able to maintain NG viability for at least 24 hours, and stabilised nucleic acids for Gen-Probe ${ }^{\circledast}$ testing. The ability to maintain culture viability of NG up to 24 hours until NAAT testing is completed could eliminate the necessity to perform cultures on all patients who are being screened for NG, with cultures maintained only for susceptibility testing for NAAT + patients.

\section{P5.070 DIAGNOSIS OF PHARYNGEAL AND RECTAL NEISSERIA GONORRHOEAE INFECTIONS}

doi:10.1136/sextrans-2013-051184.1114

'A Marangoni, ${ }^{1} \mathrm{P}$ Nardini, ${ }^{1} \mathrm{M}$ Compri, ${ }^{1} \mathrm{C}$ Foschi, ${ }^{2} \mathrm{~A}$ D'Antuono, ${ }^{2} \mathrm{~A}$ Filippini, ${ }^{2} \mathrm{C}$ Baraldi, ${ }^{2} \mathrm{C}$ Baraldi, 'R Cevenini. 'University of Bologna, Microbiology, DIMES, Bologna, Italy; ${ }^{2}$ University of Bologna, Dermatology, DIMES, Bologna, Italy

Background Despite nucleic acid amplification tests (NAAT) are widely used to detect Neisseria gonorrhoeae infections, so far no commercial kit has been cleared for testing rectal or pharyngeal swab samples, even if anal and/or oral sex practises are common.

In this study, a comparison between Real Time PCR Versant CT/ GC DNA 1.0 (Siemens) and N. gonorrhoeae culture performances has been conducted, testing rectal or pharyngeal secretions collected by E-swabs (Copan).

Methods Study group. A prospective study was performed with 171 subjects (130 males and 41 females) attending the STD Outpatients Clinic of St. Orsola Hospital, Bologna. All the patients were enrolled because having unsafe receptive anal and/or pharyngeal sex intercourses.

NAAT methods. All the specimens were tested by Versant CT/ GC DNA 1.0. As a confirmation, all the specimens scored positive for $N$. gonorrhoeae were retested, using the same extraction, by a "home-made" PCR assay, targeting cppB gene.

N. gonorrhoeae culture. Bacteria were isolated in Thayer-Martin medium and identified by API NH assay (bioMérieux). Antimicrobial susceptibility was assessed by Kirby-Bauer Test.

Results A total of 227 samples were obtained. In particular, 56 patients provided both the specimens, 89 patients provided only pharyngeal swabs, whereas only rectal specimens were collected from the remaining 26 patients.

Versant CT/GC DNA 1.0 gave positive results for $N$. gonorrhoeae in 13 pharyngeal in 7 rectal samples, all from MSM. All the Versant reactive results were confirmed by "home-made" PCR. Prevalence of rectal infection was $8.5 \%$ (7 positive out of 82 patients), whereas prevalence of pharyngeal infection was $9.0 \%$ (13/145). Culture was far less sensitive than NAAT, since only 4 samples were identified. All of them were resistant to quinolones, but susceptible to cephalosporins (cefixime and ceftriaxone).

Conclusions Pharyngeal and/or rectal screening for gonorrhoea should be considered essential in consultations for MSM in STD clinics.

\section{P5.071 EVALUATION OF ELECSYS ${ }^{\circledR}$ IMMUNOASSAY SYSTEM FOR DETERMINATION OF TYPE-SPECIFIC IGG ANTIBODIES TO HSV-1 AND HSV-2}

doi:10.1136/sextrans-2013-051184.1115 